\title{
SISTEMA COMPATIBLE DE CRECIMIENTO Y RENDIMIENTO PARA RODALES COETÁNEOS DE Pinus patula
}

\section{COMPATIBLE GROWTH AND YIELD SYSTEM FOR Pinus patula EVEN-AGED STANDS}

\author{
Wenceslao Santiago-García ${ }^{1}$, Héctor M. de los Santos-Posadas ${ }^{1 \star}$, Gregorio Ángeles-Pérez ${ }^{1}$, \\ José René Valdez-Lazalde ${ }^{1}$ y Gustavo Ramírez-Valverde ${ }^{2}$
}

\begin{abstract}
${ }^{1}$ Postgrado Forestal y ${ }^{2}$ Postgrado en Estadística, Campus Montecillo, Colegio de Postgraduados. km 36.5 Carretera México-Texcoco. 56230, Montecillo, Texcoco, Edo. de México.

*Autor para correspondencia (hmsantos@colpos.mx)
\end{abstract}

\section{RESUMEN}

\begin{abstract}
Se desarrolló un modelo de crecimiento y rendimiento para Pinus patula con el enfoque de modelos explícitos de totalidad del rodal. En su construcción se utilizó información dasométrica derivada de tres remediciones en 42 parcelas permanentes de muestreo de 400 $\mathrm{m}^{2}$, en una crono-secuencia en Zacualtipán, Hidalgo, México. La productividad de los rodales se estimó mediante un patrón anamórfico de crecimiento, basado en el modelo de Hossfeld IV e interrelacionado con las ecuaciones de área basal, diámetro promedio y volumen total de fuste por hectárea, con la técnica de regresión aparentemente no relacionada. La mortalidad se ajustó a un modelo exponencial simple, con el método de mínimos cuadrados no lineales. Al simular el escenario de productividad del sitio promedio, el modelo obtenido detectó un periodo (turno técnico) para la maximización de volumen total de madera en rollo por hectárea de 25 años, lo que contrasta con el turno empírico de 50 años utilizado en la zona desde 1980.
\end{abstract}

Palabras clave: Pinus patula, diferencia algebraica, modelos compatibles, predicción explícita.

\section{SUMMARY}

The growth and yield system for Pinus patula was developed using explicit whole-stand-approach models. The data was obtained from three consecutive re-measurements of 42 permanent plots, $400 \mathrm{~m}^{2} \mathrm{each}$, established on a chrono sequence at Zacualtipán, Hidalgo, México. Forest productivity was estimated using an anamorphic dominant height family based on the Hossfeld IV model and linked to equations of basal area, mean diameter, and total volume, with a seemingly unrelated regression fitting technique. Mortality was fitted to a simple exponential mortality function through non-linear least squares method. When simulating the scenario of site average productivity, the attained model estimated a 25 -year rotation for maximizing total volume, which contrasts with the empirical 50-year rotation used in the region since 1980 .

Index words: Pinus patula, algebraic difference, compatible models, explicit prediction.

\section{INTRODUCCIÓN}

Para lograr un manejo forestal maderable eficiente y con miras a la sustentabilidad, es necesario que los silvicultores cuenten con herramientas cuantitativas que les permitan simular y predecir los efectos posteriores del manejo que han aplicado o aplicarían. En bosques sometidos a un manejo convencional es importante contar con modelos de crecimiento promedio que describan el curso de variables del rodal (área forestal) en el tiempo. Sin embargo, en bosques bajo manejo intensivo es necesario contar con modelos de predicción que involucren una amplia variedad de condiciones de manejo (García, 1994; Vanclay, 1994; Davis et al., 2001).

En México, el avance en el desarrollo de sistemas de crecimiento y rendimiento (SCRM) ha tomado fuerza en los últimos años, sobre todo para masas naturales coetáneas y para plantaciones cuyo fin es la producción maderera (Zepeda y Domínguez, 1998; De la Fuente et al., 1998; ValdezLazalde y Lynch, 2000; Zepeda y Acosta, 2000; Maldonado y Návar, 2002; Galán et al., 2008; Magaña et al., 2008).

Un SCRM es un conjunto de ecuaciones que describe cuantitativamente el crecimiento forestal a través de años, que permite estimar los cambios en diversas variables de interés, como altura dominante, diámetro, área basal, número de árboles y volumen maderable. Cada ecuación en el sistema describe una relación diferente entre un conjunto de variables, bajo el supuesto de que todas las relaciones ocurren simultáneamente (Borders y Bailey, 1986).

Con base en el grupo de relaciones simultáneas entre variables, los SCRM se pueden clasificar en compatibles o no compatibles. Los modelos compatibles de crecimiento y rendimiento maderable comenzaron a desarrollarse a partir de la década de 1960, con los trabajos de Buckman (1962) y Clutter (1963), quienes establecieron que los componentes del crecimiento están relacionados entre sí; además, determinaron que la integral de la curva que describe la tasa de crecimiento es igual al rendimiento alcanzado en un intervalo de tiempo (Borders y Bailey, 1986; Borders, 1989). Como alternativa se inició el desarrollo de ecuaciones de diferencia algebraica para generar SCRM compatibles, también llamadas funciones de transición, que describen las tasas de cambio del sistema en cualquier punto del tiempo, en función de su estado actual (Ramírez et al., 1987; García, 1988; García, 1994). 
El objetivo de esta investigación fue desarrollar un SCRM compatible, bajo el enfoque de modelado explícito a nivel de rodal, para rodales coetáneos de Pinus patula manejados con el método de desarrollo silvícola (MDS) (HernándezDíaz et al., 2008) en la región de Zacualtipán, Hidalgo, México. Este SCRM, además de servir como herramienta para la planificación forestal, permite calcular el turno técnico en volumen (la edad óptima de cosecha para maximizar el volumen total) para la especie en la región.

\section{MATERIALES Y MÉTODOS}

\section{Área de estudio}

El estudio se hizo al sureste del municipio de Zacualtipán de Ángeles, en el Estado de Hidalgo, México, en la provincia fisiográfica Sierra Madre Oriental y Subprovincia Sierra de Zacualtipán. Se encuentra en la vertiente del Golfo de México, entre las coordenadas geográficas $20^{\circ} 36^{\prime} 44^{\prime \prime}$ y $20^{\circ}$ $37^{\prime} 49^{\prime \prime}$ LN y $98^{\circ} 35^{\prime} 30^{\prime \prime}$ y $98^{\circ} 37^{\prime} 46^{\prime \prime}$ LO, con una altitud media de $2060 \mathrm{~m}$. El suelo de las partes bajas es Feozem háplico (Hh), con una capa superficial oscura, suave y rica en materia orgánica, mientras que en las partes con mayor pendiente se encuentra el Regosol calcárico (Rc). El clima es $\mathrm{C}(\mathrm{fm}) \mathrm{w}$ ” b(e)g, es decir, templado-húmedo con lluvia todo el año, con temperatura media anual de $13.5^{\circ} \mathrm{C}$ y precipitación anual de $2050 \mathrm{~mm}$. En las últimas tres décadas, la masa forestal del área de estudio se ha orientado hacia el desarrollo de rodales coetáneos de Pinus patula (Aguirre -Salado et al., 2009).

\section{Datos dasométricos}

Los datos utilizados en este estudio se colectaron en tres inventarios efectuados en 42 parcelas permanentes de muestreo de $400 \mathrm{~m}^{2}$ cada una, las cuales cubren diferentes rangos de densidad, calidades de sitio y edades de rodales puros y coetáneos de Pinus patula. La información dasométrica consistió en: altura total (m) de todos los árboles, de los cuales cuatro se etiquetaron como dominantes $(H D)$, porque la definición de altura dominante corresponde a los 100 árboles más altos por hectárea (Alder, 1980); diámetro normal de todos los árboles $(\mathrm{DN}, \mathrm{cm})$; y número de árboles vivos $(N A)$. Las variables de estado (las variables para caracterizar una condición de crecimiento particular de un rodal) estimadas por hectárea, a partir de estos datos, fueron: altura promedio de los árboles dominantes $(H D, \mathrm{~m})$, diámetro promedio $(D P, \mathrm{~cm})$, área basal $\left(A B, \mathrm{~m}^{2}\right)$, volumen total $\left(V, \mathrm{~m}^{3}\right)$ y número de árboles vivos $(N A)$.

\section{Desarrollo de modelos compatibles}

El SCRM compatible se conforma de dos tipos de funciones: ecuaciones de predicción y sus correspondientes funciones de proyección para las variables de estado: $H D$, $A B, D P$ y $V$. El ajuste de las ecuaciones para calcular altura dominante $(H D)$ e índice de sitio (IS, se refiere a la altura dominante del rodal a una edad de referencia) es generalmente el primer paso para crear un SCRM, ya que a partir de estas ecuaciones se deduce la fertilidad o calidad de estación del bosque o rodal. Para ajustar los modelos de predicción y proyección en $H D$ se utilizaron 84 pares de datos no traslapados, correspondientes al promedio de alturas dominantes y edades que van de 8 a 27 años.

En general, el modelo de diferencia algebraica que define la familia de curvas de $I S$ tiene la forma: $Y_{2}=f\left(Y_{1}, E_{2}, E_{1}\right.$, $\beta$ ), donde $Y_{2}$ es el valor de la variable analizada del rodal a una edad $E_{2}$ (edad de proyección), $Y_{1}$ es la variable de interés medida a una edad $E_{1}$ (edad inicial) y $\beta$ es el vector de parámetros de regresión (Clutter et al., 1983; Diéguez et al., 2005; Magaña et al., 2008). Para la función $f($.) se consideraron dos hipótesis de crecimiento anamórficas y una polimórfica (Cuadro 1). La hipótesis básica de las curvas anamórficas, es que las tasas relativas de crecimiento en $H D$ entre sitios es constante pero su potencialidad máxima varía (Clutter et al., 1983). Por su parte, las curvas polimórficas se basan en la hipótesis de que la tasa relativa de crecimiento no es constante para todos los sitios. Sin embargo, la potencialidad máxima es única (Avery y Burkhart, 1983). Se debe tener en cuenta que el patrón de crecimiento en altura dominante afectará a todo el sistema, en el momento en que $H D$ o IS se utilicen como variables independientes en cualquiera de los modelos del SCRM. Por ello, la elección de sus valores no es trivial.

El paso inicial para la construcción de los modelos compatibles de área basal, diámetro promedio y volumen total consistió en definir las ecuaciones de predicción para caracterizar el estado promedio del rodal. En este caso, las ecuaciones se basaron en modificaciones del modelo básico de rendimiento de Schumacher (1939) y Clutter (1963). El segundo paso fue obtener un modelo compatible que permitiera hacer una distinción entre los modelos de predicción para el estado actual $\left(E_{1}\right)$ y los modelos de proyección para el estado futuro del rodal $\left(E_{2}\right)$. La forma de obtener un modelo compatible se ejemplifica a continuación, para el área basal, cuya ecuación de predicción es:

$$
A B_{1}=\exp \left[\alpha_{0}+\alpha_{1} \times E_{1}+\alpha_{2} \times \frac{1}{D P_{1}}\right] \times\left[I S \times N A_{1}\right]^{\alpha_{3}}
$$

donde $A B_{1}$ es el área basal $\left(\mathrm{m}^{2} \mathrm{ha}^{-1}\right)$ a la edad $E_{1} ; D P_{1}$ es el diámetro promedio $(\mathrm{cm})$ a la edad $E_{1} ; N A_{1}$ es el número de árboles $\left(h^{-1}\right)$ a la edad $E_{1} ; \alpha_{i}$ son los parámetros a estimar en el modelo. 
Cuadro 1. Estructuras de los modelos compatibles de altura dominante.

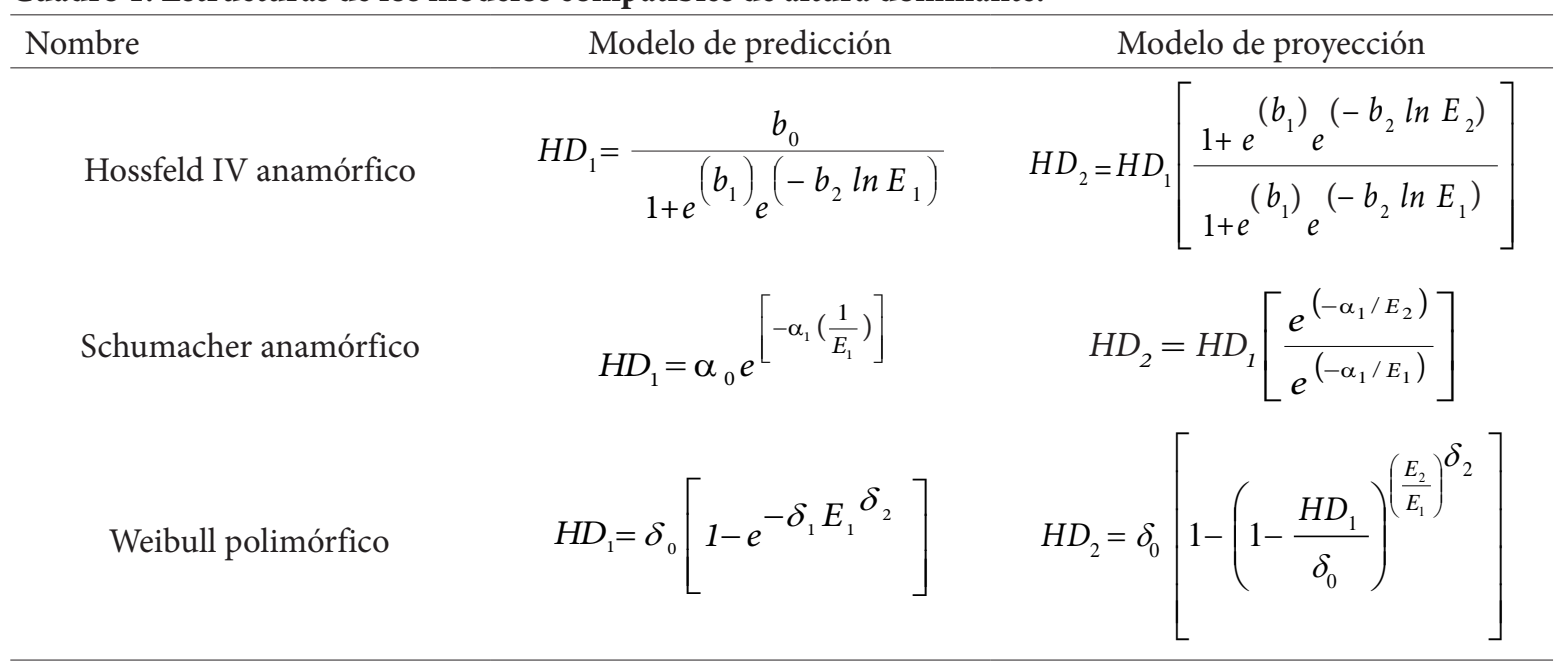

$H D_{1}$ y $H D_{2}=$ alturas dominantes en $E_{1}$ y $E_{2} ; \ln =$ logaritmo natural; $e=$ función exponencial; $b_{i}, \alpha_{i}$ y $\delta_{\mathrm{i}}=$ parámetros a estimar.

Si se lineariza la Ec. 1 para los estados $E_{1}$ y $E_{2}$, se obtienen las siguientes ecuaciones:

$$
\begin{aligned}
& \ln \left(A B_{1}\right)=\alpha_{0}+\alpha_{1} \times \mathrm{E}_{1}+\alpha_{2} \times \frac{1}{D P_{1}}+\alpha_{3} \ln \left[I S \times N A_{1}\right] \\
& \ln \left(\mathrm{AB}_{2}\right)=\alpha_{0}+\alpha_{1} \times \mathrm{E}_{2}+\alpha_{2} \times \frac{1}{\mathrm{DP}_{2}}+\alpha_{3} \ln \left[I S \times N A_{2}\right]
\end{aligned}
$$

donde $A B_{2}$ es el área basal $\left(\mathrm{m}^{2} \mathrm{ha}^{-1}\right)$ a la edad $E_{2} ; D P_{2}$ es el diámetro promedio $(\mathrm{cm})$ a la edad $E_{2} ; \mathrm{NA}_{2}$ es el número de árboles $\left(\mathrm{ha}^{-1}\right)$ a la edad $E_{2}$; los demás términos se definieron antes.

Al diferenciar ambos lados y despejar $\ln \left(A B_{2}\right)$ se obtiene:

$$
\begin{aligned}
\ln \left(A B_{2}\right) & =\ln \left(A B_{1}\right)+\alpha_{1}\left(E_{2}-E_{1}\right) \\
& +\alpha_{2}\left(\frac{1}{D P_{2}}-\frac{1}{D P_{1}}\right) \\
& +\alpha_{3} \ln \left[\frac{N A_{2}}{N A_{1}}\right]
\end{aligned}
$$

Esta última ecuación es la forma lineal del modelo de proyección para el logaritmo de $A B$. Si se aplica la función exponencial en ambos lados de la ecuación, se obtiene el modelo no lineal de proyección siguiente:

$$
\begin{aligned}
A B_{2} & =\exp \left[\ln \left(A B_{1}\right)+\alpha_{1}\left(E_{2}-E_{1}\right)\right. \\
& \left.+\alpha_{2}\left(\frac{1}{D P_{2}}-\frac{1}{D P_{1}}\right)+\alpha_{3} \ln \left(\frac{N A_{2}}{N A_{1}}\right)\right]
\end{aligned}
$$

La presencia y combinación de variables de predicción como $D P_{\mathrm{i}}$ y $N A_{\mathrm{i}}$ en la Ec. 2, requiere de ecuaciones que las definan y sean parte del SCRM. Así, para el $D P_{i}$ se propone el siguiente par de ecuaciones compatibles de predicción y proyección:

$$
D P_{1}=\exp \left[b_{0}+\frac{b_{1}}{E_{1}} \times N A_{1}+b_{2} \times H D_{1}\right]
$$

$D P_{2}=D P_{1} \times \exp \left[b_{1}\left(\frac{N A_{2}}{E_{2}}-\frac{N A_{1}}{E_{1}}\right)+b_{2}\left(H D_{2}-H D_{1}\right)\right]$

donde: $b_{\mathrm{i}}$ son los parámetros a estimar, y los demás términos se definieron antes.

Para el volumen total se proponen las siguientes ecuaciones compatibles:

$$
V_{1}=\exp \left[\delta_{0}+\frac{\delta_{1}}{A B_{1}} \times H D_{1}\right] \times E_{1}^{\delta_{2}} \times H D_{1}
$$

$$
V_{2}=V_{1} \times\left(\frac{E_{2}}{E_{1}}\right)^{\delta_{2}} \times\left(\frac{H D_{2}}{H D_{1}}\right) \times \exp \left[\delta_{1}\left(\frac{H D_{2}}{A B_{2}}-\frac{H D_{1}}{A B_{1}}\right)\right]
$$

donde $V_{1}$ es el volumen $\left(\mathrm{m}^{3} \mathrm{ha}^{-1}\right)$ a la edad $E_{1} ; V_{2}$ es el volumen $\left(\mathrm{m}^{3} \mathrm{ha}^{-1}\right)$ a la edad $E_{2} ; d_{\mathrm{i}}$ son los parámetros a estimar. 
Como parte esencial de la estimación del crecimiento y rendimiento maderable, a nivel de unidad de superficie es necesario hacer pronósticos del número de árboles sobrevivientes en un tiempo determinado. Para tal propósito se ajustó la siguiente ecuación de proyección (Torres y Magaña, 2001):

$$
N A_{2}=N A_{1} \times \exp \left[\alpha_{1}\left(E_{2}-E_{1}\right)\right]
$$

\section{Ajuste del sistema}

Cada componente del SCRM se ajustó de manera simultánea, mediante la técnica de regresión aparentemente no relacionada (SUR, por sus siglas en inglés), con el paquete Statistical Analysis System (SAS®), con excepción de la función de mortalidad que se ajustó mediante la técnica de mínimos cuadrados no lineales. Los criterios estadísticos de bondad de ajuste de los modelos fueron: suma de cuadrados del error (SCE), cuadrado medio del error (CME), y coeficiente de determinación del modelo $\left(\mathrm{R}^{2}\right)$.

La técnica SUR proporciona una mejor estimación de parámetros cuando los componentes del error se correlacionan en un sistema de ecuaciones; además, permite compatibilidad total entre el modelo de predicción y el de proyección, de forma que los parámetros comunes de ambas ecuaciones toman los mismos valores mientras se cumple con el criterio de minimización de cuadrados de los residuos (Galán et al., 2008). Los modelos descritos son intrínsecamente no lineales, por lo que la estimación de los parámetros requiere de un proceso iterativo (Diéguez et al., 2005); en este caso, se utilizó el algoritmo de GaussNewton del procedimiento MODEL de SAS/ETS (SAS Institute, 2011).

\section{RESULTADOS}

\section{Estimación de la productividad}

Los criterios estadísticos de bondad de ajuste y los parámetros estimados de las ecuaciones de crecimiento en altura dominante ajustadas de forma simultánea se presentan en los Cuadros 2 y 3, respectivamente. Los modelos generaron buenos ajustes, al explicar $93.3 \%$ (predicción) y 96.8 $\%$ (proyección) de la variación total observada en la altura dominante, y se obtuvieron valores similares en los indicadores estadísticos para los modelos propuestos en la suma de cuadrados del error (SCE), cuadrado medio del error (CME) y errores estándar reducidos.

El análisis estadístico muestra que el modelo anamórfico de Hossfeld IV resultó superior, porque presentó coeficientes de determinación $\left(\mathrm{R}^{2}\right)$ ligeramente más altos para las ecuaciones de predicción y proyección, y sobre todo porque tuvo los valores más pequeños en SCE y CME (Cuadro 2). Con base en estos criterios y en la evaluación gráfica de su comportamiento respecto a la trayectoria de los datos de campo, se eligió este modelo para la construcción de las curvas de crecimiento en altura dominante (Figura 1).

El buen comportamiento de la familia de curvas anamórficas para describir el patrón de crecimiento también se consideró como factor para la selección de la familia anamórfica de Hossfeld IV. Con una edad base $\left(E_{B}\right)$ de 40 años, se obtuvo la ecuación que expresa el índice de sitio (IS):

$$
\begin{aligned}
I S & =H D \times\left[\frac{1+e^{(4.2347)} e^{(-1.3958 \times \ln E)}}{1+e^{(4.2347)} e^{(-1.3958 \times \ln 40)}}\right] \\
& =H D \times\left[\frac{1+e^{(4.2347)} e^{(-1.3958 \times \ln E)}}{1.400822}\right]
\end{aligned}
$$

El IS permite al administrador forestal clasificar y proyectar el crecimiento del rodal, lo que representa un medio eficaz y simplificado para evaluar la productividad forestal (Clutter et al., 1983; García et al., 1998; Torres y Magaña, 2001; De los Santos-Posadas et al., 2006). Estas ecuaciones compatibles permiten: 1) Obtener la calificación de productividad al sustituir en ellas la $H D$ observada durante el inventario en $H D_{1}$, la edad del rodal por $E_{1}$ y la edad base por $E_{2}$, como sugiere la Ec. 8; y 2) Proyectar el crecimiento

Cuadro 2. Estadísticas de bondad de ajuste de los modelos de predicción y proyección del crecimiento en altura dominante.

\begin{tabular}{lcccccccc}
\hline \multirow{2}{*}{ Modelo } & \multicolumn{3}{c}{ Predicción } & & \multicolumn{3}{c}{ Proyección } \\
\cline { 2 - 3 } \cline { 7 - 8 } & SCE & CME & $\mathrm{R}^{2}$ & & SCE & CME & $\mathrm{R}^{2}$ \\
\hline Hossfeld IV anamórfico & 100.8 & 1.229 & & 0.934 & & 46.579 & 0.561 & 0.968 \\
Schumacher anamórfico & 102.4 & 1.241 & & 0.933 & & 47.385 & 0.567 & 0.967 \\
Weibull polimórfico & 102.4 & 1.249 & 0.933 & & 47.878 & 0.576 & 0.967 \\
\hline
\end{tabular}

SCE = suma de cuadrados del error; $\mathrm{CME}$ = cuadrado medio del error; $\mathrm{R}^{2}$ = coeficiente de determinación del modelo. 


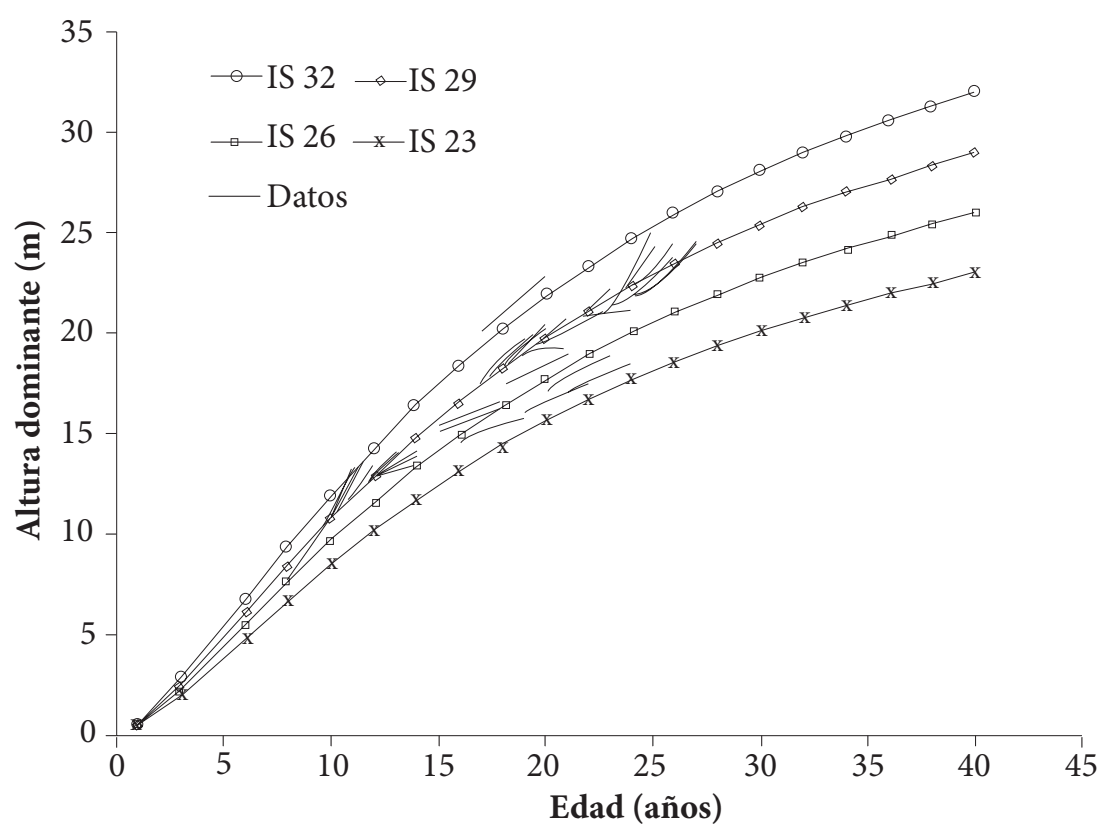

Figura 1. Curvas anamórficas de índice de sitio para Pinus patula de Zacualtipán, Hidalgo, a la edad base de 40 años (sobrepuestas a las trayectorias de los datos observados en parcelas permanentes de muestreo). IS = índice de sitio.

en $H D$ al sustituir directamente el IS y la edad base en $H D_{1}$ y $E_{1}$, respectivamente, y la edad de proyección por $E_{2}$.

\section{Ajuste de las ecuaciones compatibles}

La obtención de las ecuaciones de HD e IS permitió continuar con el desarrollo del SCRM, ya que fueron de utilidad para explicar el crecimiento en $D P, A B$ y $V$. El ajuste y los parámetros estimados para el resto de las ecuaciones que integran el SCRM muestran una precisión alta, que explica de 88.4 a 99.7 \% la variación total observada en las variables de interés (Cuadro 4). La alta significancia de los parámetros $(\mathrm{P}<0.0001)$ y sus pequeños errores estándar hacen robustos y confiables los modelos de crecimiento propuestos (Cuadro 5). De éstos, los modelos de proyección destacan por presentar SCE y CME más bajos, así como un $\mathrm{R}^{2}$ más alto que los modelos de predicción.

Al incluirse el número de árboles por hectárea (NA) como variable de predicción, se da al sistema la connotación de "densidad variable", propiedad ideal para simular regímenes silviculturales etiquetados para distintos IS. Otra cualidad que destaca en este tipo de modelos es la transitividad, es decir, los valores pronosticados de las variables de estado del rodal a un instante futuro a partir de un estado inicial deben ser iguales, independientemente del número de pasos utilizados en su predicción (Diéguez et al., 2005). García (1994) definió esta cualidad como composición o propiedad de semigrupo, esto es, el resultado de proyectar el estado, primero de $E_{0}$ a $E_{1}$ y luego de $E_{1}$ a $E_{2}$, debe ser el mismo que el de la proyección en una sola etapa de $E_{0}$ a $E_{2}$.

\section{Uso del sistema compatible}

El uso de las ecuaciones del sistema permite representar, en términos numéricos, la vida de un rodal mediante una tabla de rendimiento, la cual muestra el cambio en la magnitud de cada una de las variables dasométricas de interés a medida que cambia su edad. Sin embargo, el SCRM propuesto representa múltiples tablas de rendimiento e innumerables combinaciones para el manejo de la densidad. El Cuadro 6 presenta una tabla de rendimiento de densidad variable, para los rodales de Pinus patula en los IS más comunes del estudio que están entre los 26 y $29 \mathrm{~m}$ de altura dominante $(H D)$.

Al cambiar la densidad del rodal, la tabla de rendimiento muestra para cada calidad de sitio el crecimiento esperado en diámetro promedio y, en consecuencia, la edad a la que se alcanza cierta dimensión de interés para la industria forestal, lo que podría denominarse "turno industrial". La calidad de sitio permite distinguir varios niveles de incremento corriente anual (ICA) y de incremento medio anual (IMA) en volumen, con los que es posible definir la edad en la que ocurre el turno técnico, es decir, la edad óptima de cosecha del rodal si se desea maximizar el volumen total (la edad cuando ICA = IMA). 
Cuadro 3. Valores estimados de los parámetros para los modelos de crecimiento en altura dominante.

\begin{tabular}{lcccc}
\hline Modelo & Parámetro & Estimación & Error estándar & $\mathrm{P}>|\mathrm{t}|$ \\
\hline Hossfeld IV anamórfico & $b_{0}$ & 38.985 & 5.932 & $<.0001$ \\
& $b_{1}$ & 4.234 & 0.157 & $<.0001$ \\
\multirow{3}{*}{ Schumacher anamórfico } & $b_{2}$ & 1.395 & 0.143 & $<.0001$ \\
& $\alpha_{0}$ & 34.998 & 0.722 & $<.0001$ \\
Weibull polimórfico & $\alpha_{1}$ & -12.234 & 0.344 & $<.0001$ \\
& $\delta_{0}$ & 34.552 & 6.021 & $<.0001$ \\
& $\delta_{1}$ & 0.024 & 0.024 & $<.0001$ \\
& $\delta_{2}$ & 1.155 & 0.100 & $<.0001$ \\
\hline
\end{tabular}

Cuadro 4. Estadísticas de bondad de ajuste de los modelos de predicción y proyección en área basal, diámetro promedio, volumen y mortalidad.

\begin{tabular}{|c|c|c|c|c|c|c|}
\hline \multirow{2}{*}{ Modelo } & \multicolumn{3}{|c|}{ Predicción } & \multicolumn{3}{|c|}{ Proyección } \\
\hline & SCE & CME & $\mathrm{R}^{2}$ & SCE & CME & $\mathrm{R}^{2}$ \\
\hline Área basal & 149.9 & 2.652 & 0.924 & 18.84 & 0.327 & 0.988 \\
\hline Diámetro promedio & 126.9 & 2.226 & 0.884 & 13.10 & 0.226 & 0.987 \\
\hline Volumen & 10219.6 & 179.3 & 0.954 & 995.8 & 17.169 & 0.995 \\
\hline Mortalidad & --- & --- & --- & 376307.0 & 5085.2 & 0.997 \\
\hline
\end{tabular}

SCE = suma de cuadrados del error; $\mathrm{CME}=$ cuadrado medio del error; $\mathrm{R}^{2}=$ coeficiente de determinación del modelo.

Cuadro 5. Valores estimados de los parámetros para los modelos de crecimiento en área basal, diámetro promedio, volumen y mortalidad.

\begin{tabular}{lcccc}
\hline Modelo & Parámetros & Estimación & Error estándar & $\mathrm{P}>|\mathrm{t}|$ \\
\hline \multirow{4}{*}{ Área basal } & $\alpha_{0}$ & -3.667 & 0.330 & $<.0001$ \\
& $\alpha_{1}$ & 0.0248 & 0.002 & $<.0001$ \\
& $\alpha_{2}$ & -19.510 & 0.665 & $<.0001$ \\
& $\alpha_{3}$ & 0.743 & 0.032 & $<.0001$ \\
Diámetro promedio & & & & \\
& $b_{0}$ & 2.649 & 0.067 & $<.0001$ \\
& $b_{1}$ & -0.0048 & 0.0004 & $<.0001$ \\
Volumen & $b_{2}$ & 0.0293 & 0.0028 & $<.0001$ \\
& & & & \\
Mortalidad & $\delta_{0}$ & 2.108 & 0.104 & $<.0001$ \\
& $\delta_{1}$ & -1.478 & 0.045 & $<.0001$ \\
& $\delta_{2}$ & 0.447 & 0.029 & $<.0001$ \\
\hline
\end{tabular}


El comportamiento de los incrementos (Cuadro 6), indica que el turno técnico ocurre a los 25 años para las calidades de sitio promedio de la zona. Este resultado contrasta con el turno actual de 50 años aplicado en la región. Es probable que bajo una silvicultura intensiva y con el propósito de producir pulpa para papel, se pueda tener un turno de 20 años en los mejores sitios siempre y cuando se cumplan los siguientes dos requisitos: 1) Se promueva una rápida repoblación del rodal después de la cosecha final; y 2) Se apliquen prácticas de control de la vegetación competidora y de manejo de la densidad. En la tabla de rendimiento también es posible aproximar la respuesta del rodal a los aclareos y determinar posibilidades de corta para distintos regímenes silvícolas.

\section{Cuadro 6. Predicción del crecimiento y rendimiento maderable para los $I S$ de 26 y $29 \mathrm{~m}$, si se considera una densidad} inicial a los 10 años de 1200 árboles ha ${ }^{-1}$.

\begin{tabular}{|c|c|c|c|c|c|c|c|c|c|c|c|c|c|}
\hline \multirow{2}{*}{$E$} & \multirow{2}{*}{$N A$} & \multicolumn{2}{|c|}{$H D$} & \multicolumn{2}{|c|}{$D P$} & \multicolumn{2}{|c|}{$A B$} & \multicolumn{2}{|c|}{$V$} & \multicolumn{2}{|c|}{$I C A$} & \multicolumn{2}{|c|}{$I M A$} \\
\hline & & IS 29 & IS 26 & IS 29 & IS 26 & IS 29 & IS 26 & IS 29 & IS 26 & IS 29 & IS 26 & IS 29 & IS 26 \\
\hline 10 & 1200 & 10.8 & 9.6 & 10.8 & 10.5 & 12.9 & 11.2 & 72.2 & 62.2 & 18.8 & 16.5 & 7.2 & 6.2 \\
\hline 11 & 1160 & 11.8 & 10.6 & 12.0 & 11.6 & 15.3 & 13.3 & 91.1 & 78.7 & 18.9 & 16.5 & 8.3 & 7.2 \\
\hline 12 & 1122 & 12.9 & 11.6 & 13.1 & 12.6 & 17.6 & 15.3 & 109.3 & 94.7 & 18.3 & 16.0 & 9.1 & 7.9 \\
\hline 13 & 1084 & 13.9 & 12.5 & 14.2 & 13.6 & 19.7 & 17.1 & 127.0 & 110.2 & 17.7 & 15.4 & 9.8 & 8.5 \\
\hline 14 & 1048 & 14.9 & 13.3 & 15.2 & 14.5 & 21.6 & 18.8 & 144.1 & 125.1 & 17.1 & 14.9 & 10.3 & 8.9 \\
\hline 15 & 1014 & 15.8 & 14.1 & 16.2 & 15.4 & 23.3 & 20.3 & 160.6 & 139.5 & 16.5 & 14.4 & 10.7 & 9.3 \\
\hline 16 & 980 & 16.6 & 14.9 & 17.1 & 16.3 & 24.9 & 21.6 & 176.5 & 153.4 & 15.9 & 13.9 & 11.0 & 9.6 \\
\hline 17 & 947 & 17.5 & 15.7 & 18.0 & 17.1 & 26.4 & 22.9 & 191.9 & 166.8 & 15.4 & 13.4 & 11.3 & 9.8 \\
\hline 18 & 916 & 18.3 & 16.4 & 18.9 & 17.9 & 27.7 & 24.1 & 206.8 & 179.8 & 14.9 & 13.0 & 11.5 & 10.0 \\
\hline 19 & 885 & 19.0 & 17.1 & 19.7 & 18.6 & 28.9 & 25.1 & 221.2 & 192.4 & 14.4 & 12.6 & 11.6 & 10.1 \\
\hline 20 & 856 & 19.8 & 17.7 & 20.5 & 19.3 & 30.0 & 26.1 & 235.2 & 204.6 & 14.0 & 12.2 & 11.8 & 10.2 \\
\hline 21 & 828 & 20.5 & 18.3 & 21.3 & 20.0 & 31.1 & 27.0 & 248.7 & 216.3 & 13.5 & 11.8 & 11.8 & 10.3 \\
\hline 22 & 800 & 21.1 & 18.9 & 22.1 & 20.7 & 32.0 & 27.9 & 261.8 & 227.7 & 13.1 & 11.4 & 11.9 & 10.4 \\
\hline 23 & 774 & 21.8 & 19.5 & 22.8 & 21.3 & 32.9 & 28.6 & 274.5 & 238.8 & 12.7 & 11.0 & 11.9 & 10.4 \\
\hline 24 & 748 & 22.3 & 20.0 & 23.4 & 21.9 & 33.7 & 29.3 & 286.8 & 249.5 & 12.3 & 10.7 & 11.9 & 10.4 \\
\hline 25 & 723 & 22.9 & 20.5 & 24.1 & 22.5 & 34.5 & 30.0 & 298.7 & 259.8 & 11.9 & 10.4 & 11.9 & 10.4 \\
\hline 26 & 699 & 23.5 & 21.0 & 24.7 & 23.0 & 35.2 & 30.6 & 310.3 & 269.9 & 11.6 & 10.1 & 11.9 & 10.4 \\
\hline 27 & 676 & 24.0 & 21.5 & 25.3 & 23.6 & 35.9 & 31.2 & 321.6 & 279.7 & 11.2 & 9.8 & 11.9 & 10.4 \\
\hline 28 & 653 & 24.5 & 21.9 & 25.9 & 24.1 & 36.5 & 31.8 & 332.5 & 289.2 & 10.9 & 9.5 & 11.9 & 10.3 \\
\hline 29 & 632 & 25.0 & 22.4 & 26.5 & 24.6 & 37.1 & 32.3 & 343.1 & 298.4 & 10.6 & 9.2 & 11.8 & 10.3 \\
\hline 30 & 611 & 25.4 & 22.8 & 27.0 & 25.0 & 37.6 & 32.7 & 353.4 & 307.4 & 10.3 & 9.0 & 11.8 & 10.2 \\
\hline 31 & 590 & 25.8 & 23.2 & 27.6 & 25.5 & 38.1 & 33.2 & 363.5 & 316.2 & 10.1 & 8.7 & 11.7 & 10.2 \\
\hline 32 & 571 & 26.3 & 23.5 & 28.1 & 25.9 & 38.6 & 33.6 & 373.3 & 324.7 & 9.8 & 8.5 & 11.7 & 10.1 \\
\hline 33 & 552 & 26.7 & 23.9 & 28.5 & 26.3 & 39.1 & 34.0 & 382.8 & 333.0 & 9.5 & 8.3 & 11.6 & 10.1 \\
\hline 34 & 533 & 27.0 & 24.2 & 29.0 & 26.7 & 39.5 & 34.4 & 392.1 & 341.1 & 9.3 & 8.1 & 11.5 & 10.0 \\
\hline 35 & 516 & 27.4 & 24.6 & 29.4 & 27.1 & 39.9 & 34.7 & 401.2 & 349.0 & 9.1 & 7.9 & 11.5 & 10.0 \\
\hline 36 & 499 & 27.7 & 24.9 & 29.9 & 27.5 & 40.2 & 35.0 & 410.1 & 356.7 & 8.9 & 7.7 & 11.4 & 9.9 \\
\hline 37 & 482 & 28.1 & 25.2 & 30.3 & 27.8 & 40.6 & 35.3 & 418.8 & 364.2 & 8.7 & 7.5 & 11.3 & 9.8 \\
\hline 38 & 466 & 28.4 & 25.5 & 30.7 & 28.2 & 40.9 & 35.6 & 427.2 & 371.5 & 8.5 & 7.4 & 11.2 & 9.8 \\
\hline 39 & 451 & 28.7 & 25.7 & 31.1 & 28.5 & 41.2 & 35.9 & 435.5 & 378.7 & 8.3 & 7.2 & 11.2 & 9.7 \\
\hline 40 & 436 & 29.0 & 26.0 & 31.5 & 28.8 & 41.5 & 36.2 & 443.6 & 385.8 & 8.1 & 7.0 & 11.1 & 9.6 \\
\hline
\end{tabular}

$E=$ edad del rodal; $N A=$ número de árboles por hectárea; $H D=$ altura dominante; $I S=$ índice de sitio; $D P=$ diámetro promedio del rodal; $A B=$ área basal por hectárea; $V=$ volumen por hectárea; $I C A=$ incremento corriente anual; $I M A=$ incremento medio anual. 
Una función soslayada y básica de un SCRM es que también puede proveer actualizaciones del inventario, cuando se carece de remediciones en la red de sitios permanentes diseñada para ello. Según la calidad y el tiempo entre remediciones, el SCRM permite: 1) Estimar un nuevo inventario a partir de uno previo, o 2) A partir de una actualización de este tipo, estimar el nuevo tamaño de muestra en caso de que se requiera un nuevo inventario operativo.

\section{DISCUSIÓN}

La información dasométrica de sitios permanentes de muestreo permitió aplicar el método de desarrollo de curvas de diferencia algebraica. Al ajustar de manera simultánea las ecuaciones de predicción y proyección, se logró la compatibilidad del SCRM. Al respecto, Zepeda y Domínguez (1998) y Zepeda y Acosta (2000) señalaron que el crecimiento y el rendimiento maderable deben ser modelados matemáticamente con la mayor precisión posible, por lo que debe existir compatibilidad analítica manifiesta entre las funciones de un SCRM, como ocurre en este caso.

El nivel de precisión del SCRM concuerda con lo reportado por Galán et al. (2008), quienes con base en datos de sitios con remediciones y mediante regresión aparentemente no relacionada, desarrollaron un sistema compatible para Cedrela odorata L. y Tabebuia donnell-smithii Rose, cuya precisión explica de 89.6 a $99.6 \%$ de la variabilidad total observada en altura dominante, área basal y volumen. Niveles similares de precisión fueron obtenidos por Magaña et al. (2008), con un sistema de ecuaciones para predecir el crecimiento y rendimiento de Pinus rudis Endl. en Aloapan, Oaxaca, con base en datos de la misma naturaleza. Los resultados también son similares a los reportados por De la Fuente et al. (1998), quienes propusieron modelos de predicción del crecimiento y rendimiento para rodales coetáneos de Pinus rudis Endl. en Pueblos Mancomunados, Ixtlán, Oaxaca. Sin embargo, estos autores utilizaron datos provenientes de parcelas temporales de muestreo.

El modelo de predicción de área basal (Ec. 1) considera como variables de predicción a: la edad del rodal, el número de árboles/ha, el índice de sitio y el diámetro promedio del rodal. Por su parte, su correspondiente modelo de proyección (Ec. 2) excluye al índice de sitio por el hecho de ajustar el sistema de forma simultánea, lo que concuerda con los resultados de otras investigaciones en las que se analizó el crecimiento del área basal (Buckman, 1962; Diéguez et al., 2005), sin incorporar el índice de sitio porque no fue significativa su participación. Esto no disminuyó la capacidad predictiva del modelo, ya que el diámetro promedio del rodal depende de la calidad de sitio, y el crecimiento del área basal es superior en las mejores calidades de sitio.
Las ecuaciones de predicción y proyección en diámetro promedio a nivel de rodal (Ecs. 3 y 4 , respectivamente) fueron esenciales para estimar el área basal. El diámetro del rodal se aprecia como la variable dasométrica más sensible al efecto de la densidad, que repercute de manera importante en el crecimiento del área basal. Las funciones de crecimiento para esta variable se construyeron con base en: la edad, el número de árboles/ha y una medida de la calidad del sitio, como lo es la altura dominante; tales funciones permitieron expresar con precisión los niveles de productividad de los rodales de Pinus patula. Es decir, en los sitios con mejor calidad el arbolado tiene mayores dimensiones en sus variables dasométricas, que en los sitios de menor calidad.

Por su parte, las ecuaciones de volumen a nivel de rodal (Ecs. 5 y 6) incorporan como variables independientes a la edad, el área basal y la calidad del sitio (expresada mediante el índice de sitio o la altura dominante), variables que hacen posible la modelación del rendimiento maderable. Estas variables se han utilizado convencionalmente para modelar el rendimiento maderable de Pinus arizonica Engl. (Zepeda y Domínguez, 1998), Pinus rudis Endl. (De la Fuente et al., 1998; Magaña et al., 2008), Pinus patula (Valdez-Lazalde y Lynch, 2000), Pinus montezumae Lamb. (Zepeda y Acosta, 2000), Cedrela odorata L. y Tabebuia donnell-smithii Rose (Galán et al., 2008).

La función de mortalidad (Ec. 7) muestra resultados razonables de acuerdo con los datos observados en campo (Figura 2), con una tasa de mortandad de $3.3 \%$ anual. Esta ecuación calcula el número de árboles sobrevivientes $\left(N A_{2}\right)$ a partir del número actual de árboles $\left(N A_{1}\right)$, y la edad actual del rodal $\left(E_{1}\right)$ con base en un periodo de proyección $\left(E_{2}\right)$. El modelo concuerda parcialmente con el reportado por Magaña et al. (2008), quienes presentaron una función de mortalidad para Pinus rudis Endl. cuya tasa de mortalidad proporcional depende de la edad y el índice de sitio. Es importante destacar que para hacer pronósticos de rendimiento es necesario establecer un valor asintótico mínimo, ya que el modelo de mortalidad eventualmente calcula obtener cero árboles/ha, cuando en un bosque manejado esto sólo ocurre después de un evento catastrófico. Es decir, ese valor mínimo es la densidad mínima razonable que se puede encontrar en los rodales de cierta edad, a partir de la cual ya no habría mortalidad.

Las ecuaciones obtenidas tienen su mejor comportamiento y capacidad de predicción en el rango de 8 a 27 años de edad del rodal, por lo que pronósticos fuera de este rango deben tomarse con reservas. Es importante destacar que en la predicción del crecimiento y rendimiento se puede incurrir en una desviación del estimador respecto al parámetro que se pretende pronosticar, debido a que los errores son 


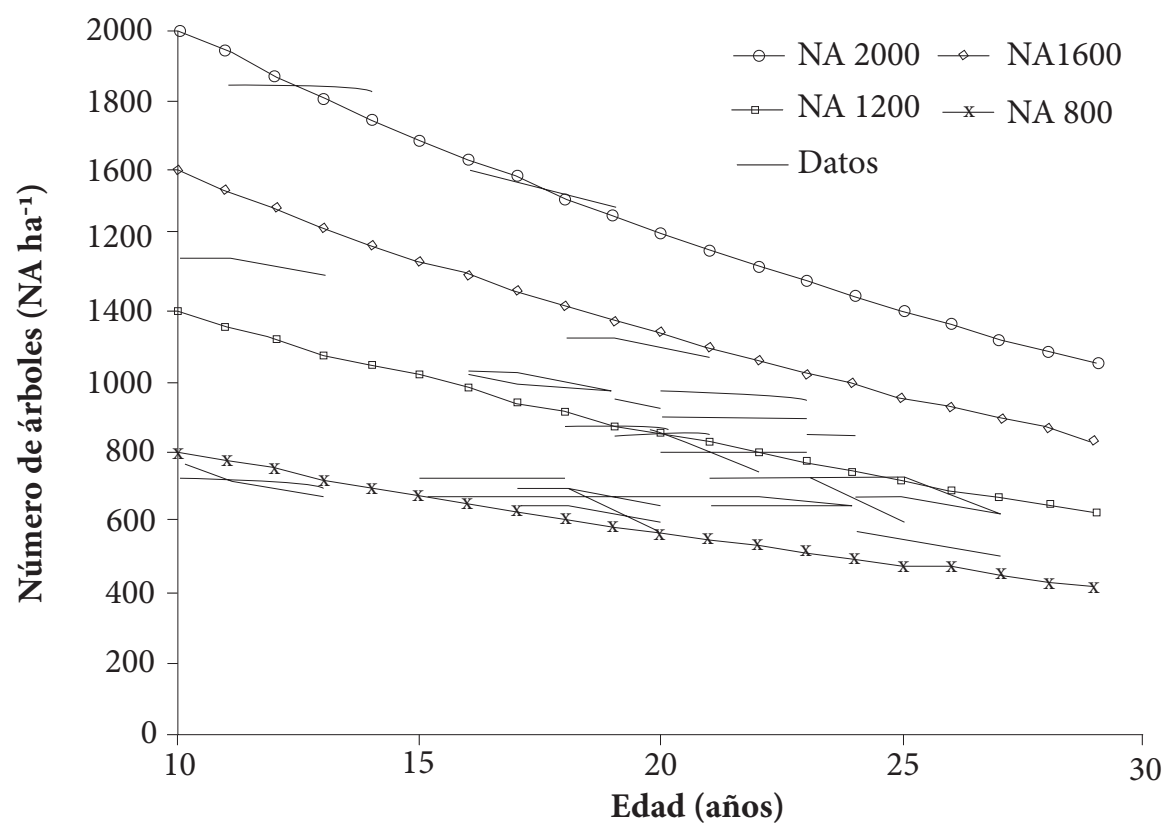

Figura 2. Sobrevivencia simulada para cuatro densidades iniciales en rodales de Pinus patula, sobrepuestas a las trayectorias de los datos observados en parcelas permanentes de muestreo. NA = número de árboles.

acumulativos conforme va interactuando el SCRM. Al respecto, Magaña et al. (2008) señalaron que una predicción confiable debe considerar una validación, tanto de cada componente como del total.

Mediciones futuras de las variables de estado del rodal permitirán ampliar el rango de validez de las ecuaciones, así como establecer nuevos patrones de crecimiento y mortalidad natural de los bosques de Pinus patula en la región de Zacualtipán, Hidalgo. Estas ecuaciones pueden incorporarse a un simulador de crecimiento en un programa computacional para representar interactivamente infinidad de escenarios en un rodal, y así modelar su crecimiento y rendimiento actual y futuro.

El sistema de crecimiento y rendimiento se puede complementar con una ecuación que permita hacer la distribución de productos/ha; para ello se requiere de un sistema ahusamiento-volumen comercial para la cubicación del arbolado. Asimismo, la modelación del crecimiento del rodal vía distribuciones diamétricas puede ser complementario al enfoque aquí presentado, que proporcionaría mayor detalle de la distribución de tamaños de árboles en un rodal, pero sería menos directo que el enfoque aquí propuesto.

\section{CONCLUSIONES}

El sistema de ecuaciones de crecimiento y rendimiento construido (modelo), permite simular un grupo amplio de escenarios silvícolas para el manejo de rodales coetáneos de
Pinus patula con el método de desarrollo silvícola (MDS) en Zacualtipán, Hidalgo. Este modelo desarrollado ya detectó que los turnos utilizados actualmente para el manejo operativo de los bosques de Pinus patula, son demasiado largos (50 años) y con intervenciones poco frecuentes. Los niveles de productividad sugieren un turno técnico promedio de 25 años, con lo cual disminuiría en 25 años la actual edad de rotación que se utiliza en los programas de manejo operativo en la región. Con el sistema de crecimiento y rendimiento modelado se obtienen predicciones y proyecciones precisas. Sin embargo, aún es necesaria la validación del modelo para que pueda ser usado en los programas de manejo y planificación de rodales coetáneos de Pinus patula en la región de estudio.

\section{AGRADECIMIENTOS}

Al fondo de investigación para la Educación SEP-CONACyT proyecto 44872, por el financiamiento parcial para esta investigación.

\section{BIBLIOGRAFÍA}

Aguirre-Salado C A, J R Valdez-Lazalde, H M De los Santos-Posadas, G Ángeles-Pérez, R Haapanen, A I Aguirre-Salado (2009) Mapeo de carbono arbóreo aéreo en bosques manejados de pino Patula en Hidalgo, México. Agrociencia 43:209-220.

Alder D (1980) Estimación del Volumen Forestal y Predicción del Rendimiento con Referencia Especial a los Trópicos. Vol. 2. Estudio FAO: Montes 22/2. Organización de las Naciones Unidas para la Agricultura y la Alimentación. Roma, Italia. 118 p.

Avery T E, H E Burkhart (1983) Forest Measurements. McGraw-Hill 
series in Forest Resources, Nueva York. USA. 331 p.

Borders B E (1989) System of equations in forest stand modeling. For. Sci. 35:548-556.

Borders B E, R L Bailey (1986) A compatible system of growth and yield equations for slash pine fitted with restricted three-stage least squares. For. Sci. 32:185-201.

Buckman R (1962) Growth and yield of red pine in Minnesota. USDA Forest Service, Tech. Bull. 1272. 50 p.

Clutter J L (1963) Compatible growth and yield models for loblolly pine. For. Sci. 9:354-371.

Clutter J L, J C Forston, L V Pienaar, G H Brister, R L Bailey (1983) Timber Management: A Quantitative Approach. John Wiley \& Sons, Inc. New York. 333 p.

Davis L S, K N Johnson, P S Bettinger, T E Howard (2001) Forest Management. 4th ed. McGraw-Hill Series in Forest Resources. 804 p.

De la Fuente E A, A Velásquez M, J M Torres R, H Ramírez M, C Rodríguez F, A Trinidad S (1998) Predicción del crecimiento y rendimiento de Pinus rudis Endl., en Pueblos Mancomunados, Ixtlán, Oaxaca. Rev. Ciencia Forestal en México 23:3-8.

De los Santos-Posadas H M, M Montero-Mata, M Kanninen (2006) Curvas dinámicas de crecimiento en altura dominante para Terminalia amazonica (Gmel.) Excell en Costa Rica, Agrociencia 40:521-532.

Diéguez A U, F Castedo D, J G Álvarez G (2005) Funciones de crecimiento en área basimétrica para masas de Pinus sylvestris L. procedentes de repoblaciones en Galicia. Invest. Agr. Sist. Recur. For. 14:253-266.

Galán L R, H M De los Santos P, J I Valdez H (2008) Crecimiento y rendimiento maderable de Cedrela odorata I. y Tabebuia donnellsmithii Rose en San José Chacalapa, Pochutla, Oaxaca. Madera y Bosques 14:65-82.

García C X, H Ramírez M, C Rodríguez F, J Jasso M, C A Ortíz S (1998) Îndice de sitio para caoba (Swietenia macrophylla King) en Quintana Roo, México. Rev. Ciencia Forestal en México 23:9-19.

García O (1988) Growth modeling-a (re)view. N. Z. For. 33:14-17.
García O (1994) The state-space approach in growth modeling. Can. J. For. Res. 24:1894-1903.

Hernández-Díaz J C, J J Corral-Rivas, A Quiñones-Chávez, J R BaconSobbe, B Vargas-Larreta (2008) Evaluación del manejo forestal regular e irregular en bosques de la Sierra Madre Occidental. Madera y Bosques 14:25-41.

Magaña T O S, J M Torres R, C Rodríguez F, H Aguirre D, A M Fierros G (2008) Predicción de la producción y rendimiento de Pinus rudis Endl. en Aloapan, Oaxaca. Madera y Bosques 14:5-19.

Maldonado D A, J Návar Ch (2002) Ajuste y predicción de la distribución Weibull a las estructuras diamétricas de plantaciones de pino de Durango, México. Madera y Bosques 8:61-72.

Ramírez M H, R L Bailey, B E Borders (1987) Some implications of the algebraic difference method approach for developing growth models. In: Forest Growth Modeling and Prediction. IUFRO. Minneapolis, USA.

SAS Institute (2011) SAS/ETS® 9.3 User's Guide. Cary, NC: SAS Institute Inc.

Schumacher F X (1939) A new growth curve and its application to timber-yield studies. J. For. 37:819-820.

Torres R J M, O S T Magaña (2001) Evaluación de Plantaciones Forestales. Centro de Investigación y Docencia Económicas A. C. Instituto Nacional de Investigaciones Forestales y Agropecuarias. Ed. Limusa, México. 472 p.

Valdez-Lazalde J R, T B Lynch (2000) Merchantable and total volume equations for thinned natural stands of patula pine. Agrociencia 34:747-758.

Vanclay J K (1994) Modeling Forest Growth and Yield, Applications to Mixed Tropical Forests. CAB International. Denmark. 312 p.

Zepeda B E M, A Domínguez P (1998) Niveles de incremento y rendimiento maderable de poblaciones naturales de Pinus arizonica Engl., de El Poleo, Chihuahua. Madera y Bosques 4:27-39.

Zepeda B E M, M Acosta M (2000) Incremento y rendimiento maderable de Pinus montezumae Lamb., en San Juan Tetla, Puebla. Madera y Bosques 6:15-27. 